\title{
La voie des chemins de fer secondaires dans les archives du ministère de l'Équipement
}

\section{Michel Bergeyre}

\section{OpenEdition}

\section{Journals}

Édition électronique

URL : https://journals.openedition.org/rhcf/2076

DOI : 10.4000/rhcf.2076

\section{Éditeur}

Rails \& histoire

\section{Édition imprimée}

Date de publication : 2 mai 2002

Pagination : 321-327

ISBN : 00996-9403

ISSN : 0996-9403

\section{Référence électronique}

Michel Bergeyre, «La voie des chemins de fer secondaires dans les archives du ministère de

I'Équipement », Revue d'histoire des chemins de fer [En ligne], 24-25 | 2002, mis en ligne le 21 avril 2015, consulté le 22 avril 2022. URL : http://journals.openedition.org/rhcf/2076 ; DOI : https://doi.org/ $10.4000 /$ rhcf.2076 


\section{La voie des chemins de fer secondaires dans les archives du ministère de l'Équipement}

Il est naturel pour nombre d'historiens ou de chercheurs, amateurs et curieux des anciens " petits trains ", après avoir fait le plein de cartes postales, de se diriger d'abord vers les archives départementales. L'inscription de ces réseaux dans l'histoire économique, voire politique, des départements, leur patrimoine immobilier subsistant et, surtout, la mémoire locale, ne les pousseront généralement pas jusqu'à Paris.

C'est ainsi que le fonds d'archives généré par l'administration centrale des ministères des Travaux publics, puis des Transports et, aujourd'hui, de l'Équipement, demeure peu exploité. Ceci est d'ailleurs vérifié pour les réseaux secondaires tout comme pour le « grand » chemin de fer, celui des compagnies puis de la SNCF.

En comparaison des grands réseaux, les lignes qui apparaissent principalement sous le vocable administratif de voies ferrées d'intérêt local, les V.F.I.L., sont pourtant relativement bien représentées tant à Paris, pour la période la plus ancienne où les versements, regroupés dans la série F14, se situent en majorité avant la Seconde Guerre mondiale, qu'à Fontainebleau, au Centre des archives contemporaines (C.A.C.) pour les documents plus récents.

La nécessité d'un contrôle accru de l'administration centrale sur une multiplicité de petites sociétés de formes diverses : régies, entreprises privées, groupes de transport, les enjeux politiques amenés lors de leur création ou de leur fermeture expliquent cette part relative. Le parlementarisme de la III république puis les difficultés précoces de ces liaisons, plus proches de la vie quotidienne d'une France encore rurale, alimentèrent de multiples soucis tandis que la proximité parisienne et le caractère quelque peu "incestueux» des relations entre fonctionnaires et dirigeants des grandes compagnies puis de la SNCF rendirent le contrôle du réseau national plus formel sinon plus lâche. 


\section{Constitution et nature des fonds}

Les versements aux Archives nationales ont été effectués de manière sporadique à l'occasion du manque de place dans les caves, souvent peu propices à la bonne conservation du papier, enfin des déménagements des services du ministère survenus entre le boulevard Saint-Germain et l'Arche de la Défense à partir de 1989. La manière d'instruire les dossiers, qui étaient des outils de travail avant de devenir des archives, a conduit pour chaque thème ou objet à de nombreux mélanges de chronologie suivant les nécessités du rappel de documents plus anciens. Les dates mentionnées sur les différents versements se chevauchent donc allègrement et la séparation entre les documents conservés à Paris et ceux présents au C.A.C. de Fontainebleau est rendue encore moins pertinente. Tout comme le faible niveau de description de la plupart des répertoires, rédigés dans l'urgence, l'amplitude et la généralité de certains thèmes, "réglementation », "gestion et comptabilité ", « travaux divers » commandent au chercheur la peine de devoir consulter une masse importante et le devoir de mesurer son effort avec son objet.

Pour illustrer ce fait, l'observation qui accompagne le versement $\mathrm{n}^{\circ} 1980-0029$ est éclairante : « Les archives concernant les V.F.I.L. ont déjà été versées à plusieurs reprises aux AN, série F14, toutefois des dossiers ont été encore retrouvés dans les caves du boulevard SaintGermain, il semble que ce soit les derniers. Les dossiers, objets du présent répertoire, sont des dossiers complets et peuvent néanmoins faire double emploi pour certaines pièces avec les dossiers précédemment envoyés. Il conviendrait de comparer avec précision, à quels tronçons de ligne ces divers dossiers ont trait. Ces dossiers, sous réserve de refonte avec F14, méritent une conservation permanente et sont, dans leur ensemble, communicables. »

Un dossier type est également décrit : «Préparation de la concession, avenants et conventions d'exploitation, cahier des charges, contrôle des tarifs de l'exploitation du point de vue financier et technique (achat de matériel, travaux), les propositions de déclassement et la mise en place des réseaux routiers de remplacement, [...] d'autre part on trouve des renseignements sur les sociétés concessionnaires, leurs fusions, leurs faillites et leur reprises. »

Le versement en question, représentant 80 articles et 11 mètres linéaires, couvre une période allant de 1875 à 1976, soit un siècle qui a vu le plein développement et la mort de la plupart des réseaux secondaires. 
Dans des versements ultérieurs, des pièces anciennes, plans et schémas des installations, rapports des ingénieurs, voire des copies des décrets octroyant les concessions au XIX ${ }^{e}$ siècle, émaillent encore les notes et correspondances adressées lors des fermetures et des déclassements progressifs intervenus ces quarante dernières années. Les contentieux surgis lors des ventes immobilières par les Domaines sont alors l'occasion de fixer avec précision les emprises des gares, des dépôts.

\section{Ressources et enseignements}

Les amateurs de matériel roulant et de technique peuvent être à première vue frustrés, les automotrices, locomotives, voitures, wagons et équipements divers n'apparaissant généralement que sous la rubrique : " achat de matériel », voire dans quelques rares rapports concernant des points précis de sécurité ou de confort: "accès ", "chauffage ", englobés dans les rubriques « exploitation », " organisation et fonctionnement ». Dans certains cas, les dates effectives de commande ou de réception des matériels y pourront être utilement recoupées quand les archives propres aux réseaux sont incomplètes ou celles des constructeurs disparues.

La valeur du sujet technique n'est pas partagée ou comprise par tous et le commentaire accompagnant le versement $n^{\circ} 1980-0028$ peut se révéler frustrant sur ce point : « Certains dossiers concernant les achats d'automotrices et autorails ont été conservés à titre de spécimen. Des plans et des photos pourront servir lors d'une exposition [...] ; Ont été conservés aussi à titre de spécimen : un dossier d'acquisition du matériel roulant du Vivarais-Lozère pendant l'année 1925. Un dossier de construction de voitures et wagons du réseau de Corse, un dossier d'acquisitions des réseaux divers de 1939.»

En revanche, pour ce même versement, les obligations émises ont rencontré plus d'enthousiasme : "Cette série présente un intérêt historique, 1897 à 1937. Le ministère des Travaux publics instruisait jusqu'à cette époque les dossiers de demande d'autorisation d'émission d'actions et d'obligations parallèlement avec le ministère des Finances et le ministère de l'Intérieur, pour le Métropolitain ; depuis la direction du Trésor est la seule à être saisie de ces demandes. » Dans les archives, le mélange arbitraire de l'histoire administrative et de l'image, pourtant exemplaire, de la technique, n'a pas été favorable à la seconde.

Le dernier versement au Centre des archives contemporaines de Fontainebleau (C.A.C.) a été effectué en 1998, n 1998-0587, articles 47 à 64, pour des documents couvrant une période allant de 1932 à 1990. Le bureau de la Réglementation générale des chemins de fer et de leur 
sécurité, au sein de la direction des Transports terrestres, sous-direction des Chemins de fer, a enregistré ainsi l'arrêt, le déclassement ou la vente par les domaines de nombre de lignes dans les années 1970 et 1980.

Les documents sont particulièrement éclairants pour les réseaux industriels d'importance comme celui des Houillères du Bassin du Nord et du Pas-de-Calais, le chemin de fer de Somain à Anzin, ceux de Chauny à Saint-Gobain, Carvin à Libercourt et de Gorcy à la frontière belge, qui jouaient de manière indépendante au grand chemin de fer: voie normale, parc et dépôt de matériel roulant conséquents, desserte de gares marchandises, service voyageurs, et dépendaient étroitement de lui pour leurs expéditions. Les dossiers y retracent les différents textes renouvelant les concessions, assortis généralement de rapports des ingénieurs, la disparition du service voyageurs et des trains ouvriers, les aliénations successives jusqu'à la vente des derniers biens et les éventuels contentieux.

Dans les archives comme dans la réalité, les deux réseaux de chemin de fer de la Provence et de la Corse y continuent leur difficile chemin ; des rapports peu enthousiastes sur l'avenir de ces lignes sont aussi nourris par les craintes engendrées par l'entretien minimal d'une infrastructure usée que rabotent encore des travaux routiers ou des opérations d'urbanisme.

Les chemins de fer touristiques font leur apparition, précoce avec la petite ligne d'Anse en 1962 dont le statut est encore à définir. Ils se heurtent à de nombreux obstacles, dont l'incrédulité de l'administration quand ce ne sont pas des dissensions internes aux associations, avec les exploitations de la vallée de la Doller ou du Rabodeau et l'expérience sans suite sur la ligne Hyères-Les Salins-d'Hyères.

Le chemin de fer de La Mure, dont l'exploitation charbonnière menaçait de s'interrompre, fait l'objet d'un article entier où les courriers se succèdent en suivant les initiatives locales. Pour la première fois une ligne de chemin de fer apparait comme un patrimoine et un atout majeur d'un petit pays enclavé pour assurer sa reconversion à travers le tourisme.

\section{Instruments de recherche}

Les versements d'archives du ministère de l'Équipement énumérés ci-dessous émanent des services chargés au niveau central de coordonner la politique des V.F.I.L., abordée sous l'angle des chemins de fer ou, parfois, des transports urbains, suivant les aléas des organigrammes. 
Versés au Centre des archives contemporaines (C.A.C.) de Fontainebleau, ils sont sommairement répertoriés jusqu'en 1995 dans l'État des versements aux Archives nationales. ${ }^{1}$. Cet ouvrage fait état de la cotation actuelle, par numéro d'entrée au C.A.C. et, pour les dossiers entrés avant 1986, d'une cotation complémentaire où le préfixe TT représente les transports terrestres.

Cet instrument de recherche est complété par des répertoires plus détaillés mais non imprimés, qui sont peu à peu intégrés dans la base des archives définitives du ministère de l'Équipement, interrogeable en plein texte par le biais de la Mission des archives auprès du ministère de l'Équipement ${ }^{2}$. La consultation de cette base peut, le cas échéant, mettre en exergue des documents émanant d'autres instances centrales de la direction des Transports terrestres ayant pu aborder la question des V.F.I.L. (cabinet, conseil général des Ponts et Chaussées...).

Enfin, les répertoires des documents versés auparavant aux Archives nationales à Paris, conservés sous la responsabilité scientifique de la Section du XIX ${ }^{e}$ siècle, et regroupés dans la sous-série F14, peuvent être consultés au Centre historique des Archives nationales (C.H.A.N.) ${ }^{3}$.

Maintenant figées, pour la période où le chemin de fer tentait de gagner sa place dans un monde encore majoritairement rural, les archives du ministère de l'Équipement offrent un complément d'information non négligeable sur un sujet qui ne peut être encore refermé malgré les études et les nombreuses publications.

Les documents qui entrent aujourd'hui dans les magasins d'archives traitent des schémas régionaux de transport et du conventionnement, du développement des services TER et du retour progressif des investissements. Ici, l'avenir du chemin de fer s'inscrit toujours dans des tracés légués par les deux derniers siècles, une continuité que refléteront également les archives.

\section{Notes}

1- Paris, La Documentation française, 1997.

2-DAFAG/MA, Tour Pascal B, Paris La Défense, pièce 23-46, téléphone 01408192 64, télécopie : 0140816408 et mél : ma.dafag@equipement.gouv.fr

3- Pour connaître les modalités de recherche et d'accès au C.H.A.N. ou au C.A.C., voir les sites Internet: http://www.archivesnationales.culture.gouv.fr/chan/ index.html ou http://www.archivesnationales.culture.gouv.fr/cac/index.html 


\title{
Tableau indicatif des versements ou articles « V.F.I.L.» conservés au Centre des archives contemporaines de Fontainebleau
}

\author{
1977-0933 art : 3-4, 7, Comité d'organisation, \\ $1939-1948$ \\ reconstitutions \\ 1977-0925 art : 1-11 Plans d'équipement, préparation et \\ $1946-1965$ \\ exécution dont Métropolitain (classé à \\ Transports urbains)
}

1977-0928 art : 3-4 Algérie, chemins de fer sur route et

$1881-1965$ V.F.I.L.

1977-0935 art : 1-10 Contrats, équipements, subvention

$1923-1963$

1977-0938 art : 1-3 Réseau corse

$1883-1955$

art : 4-10 Chemins de fer économiques

1887-1964

1977-0996 art : 1-37 Travaux et déclassements, textes

$1867-1974$ réglementaires

1977-1010 art : 1 Décret loi du 23 octobre 1935 sur la

$1935-1968$ révision des concessions et application, suppressions et déclassements

1977-1029 art : Déclassement de lignes

$1944-1974$

1980-0028 art : 1-13 Ch. de fer d'outre-mer, La Réunion

$1878-1970$

art : 14-15 Réglementation, avis du Conseil d'État

$1872-1893$

TT3625-3626

art : 16-21 demandes d'autorisation

$1891-1953$

d'émission d'actions

art : 22-23 matériel roulant

TT3633-3637

$1872-1970$

1980-0029 art : 1-5 Lois de 1913 et 1922, travaux, exploitation TT3665-3710

art : $77-80$ ch. de fer industriels

TT3711-3714

1897-1969

$1875-1966$

1980-0431 art : 1-17 Travaux, enquêtes d'utilité publique, plans

$1855-1957$ TT4070-4086 
1981-0313 art : 69 Chemins de fer touristiques, Vivarais, Bretagne $\quad$ 1965-1977 TT4337

art : 70-74 Ch. de fer secondaires, rapports annuels 1963-1978 TT4338

1983-0103 art : Tutelle des V.F.I.L. et tramways

$1846-1957$

TT5202-5245

1985-0009 art :1-4 Gestion des concessions, contrôle d'exploitation 1870-1978 TT6518-6521

1998-0587 art : 47-49 Chemin de fer de la Corse

$1968-1990$

art : 50-53 Chemin de fer de Provence, Nice Digne 1945-1990

art : 54 Déclassements, Bouches du Rhône, 1947-1987 Landes, Sarthe

art : 55-61 Ch. de fer industriels, H.B.N.P.C., 1932-1987

Somain-Anzin,...

art : 62-64 Ch.de fer touristiques, Doller, 1962-1987

Rabodeau, La Mure 